\title{
Analysis of collaborative design and decision making through argumentation applied for pre-BIM stages
}

\author{
M. Leon ${ }^{1} \&$ A. Toniolo ${ }^{2}$ \\ ${ }^{1}$ Scott Sutherland School of Architecture and the Built Environment, \\ Robert Gordon University, UK \\ ${ }^{2}$ Department of Computing Science, University of Aberdeen, UK
}

\begin{abstract}
The early design stages are the most vital for the development of the design of a building project and the decisions taken during these steps are significant for the further progress of the project, regarding aspects like cost, performance, reliability and sustainability. Being able to trace backwards the thoughts that led to these decisions at any point of the design process provides clarity and transparency during the decision making process. Additionally, monitoring the design steps and decisions can lead to further observations on the effects of the design decisions during the early design stage, thus leading to a more sustainable and holistic design approach. This paper claims that efficient monitoring of the decision steps can be achieved employing a computational model of argumentation. Argumentationbased reasoning helps identify the rationale for a decision (i.e. arguments) and the relationships (i.e. attacks and preferences) between conflicting issues involved in making decisions. Our approach provides a method to rigorously trace the resolution of conflicts by extracting the set of acceptable arguments that led to a decision, and may eventually assist designers in analysing complex collaborative decisions within a pre-Building Information Modelling context.

Keywords: collaborative design, argumentation, pre-BIM, decisions, conflicts.
\end{abstract}

\section{Introduction}

Increased multidisciplinary effort during the early design stages of the Architecture, Engineering and Construction (AEC) industry is a prerequisite 
for effective overall design and construction stages, especially due to the Building Information Modelling (BIM) mandate in 2016 [1,2]. BIM is changing collaboration processes and it is shifting the focus from the chain of activities to efficient collaboration and to innovative ways of creating, sharing and collecting relevant information among different but project related disciplines [3]. Shift of the effort towards the early and conceptual design stages has the potential to lead to fewer problems during the later more complex design steps and the most important requirement is the effective collaboration among the different professionals and disciplines.

The method suggested by Leon [1] for tackling problems with workflow [4-6], education [7] and organisation involves the development of an organised protocol that includes aspects like team building [8] and design and communication management [9]. Furthermore, design processes that have been modelled previously according to engineering [10], design [4,11,12] up to the AEC industry [13-15] have supported the synthesis, development and testing of a predefined and multidisciplinary Conceptual Design Stages Protocol (CDS Protocol) [1]. CDS Protocol supports the multi-party agreement and multidisciplinary early involvement for maximising the potentials of collaboration and coordination for the entirety of a project.

Leon [1] proposed this novel CDS Protocol to be adopted by AEC professionals during pre-BIM stages in order to address the lack of an organised system for supporting the early conceptual design stages, enhancing multidisciplinary collaboration and providing informed design solutions. The CDS-Protocol initiates with the team formation and the introduction of the brief, followed by discussion of project requirements, solution synthesis and brainstorming, solution evaluation, consensus and the final solution. Decision points identified in this process reflect the shared views and agreements among the participants regarding the project, for the purpose of moving forward the design. Feedback loops allow the reconsideration of the achieved consensus in case this informed compromise does not comply with the design brief requirements and the project objectives.

The adoption of this protocol was evaluated within a set of experimental studies involving a diverse team of professionals with experience in the AEC industry. The analysis showed that the CDS-Protocol for pre-BIM phases led to greater collaboration among the participants and enhanced cognitive and conceptual activities. The process allowed for the promotion of ideation together with advanced solutions in a reduced amount of time, and an increased satisfaction of the participants demonstrating that the CDS-Protocol makes a significant improvement of the design process and leads to more effective teamwork and communication.

Interestingly, the CDS-Protocol proposed by Leon [1] takes into consideration how the design process evolves in the light of new information introduced by the professionals, which arises from previous experiences, knowledge, expertise and design attributes. This incremental addition of information and collaborative reasoning moves the process forward to fulfil the design requirements. However, in a team context, professional opinions and requirements may be conflicting and 
new information may also be conflicting or incomplete. Domain knowledge may be not be aligned among professionals due to their different expertise and together these issues may impede to establish agreements on the design solutions.

In this research, we propose the use of argumentation-based reasoning to investigate how requirements, expertise and information flow contribute towards informed design decisions. As argued in Leon et al. [16], this is an iterative method of updating the solution space in the light of new constraints, goals and alternatives evaluation. Argumentation provides a natural way to model this process. The aim of computational models of argumentation-based reasoning is to represent and analyse positive and negative reasons (i.e., pro and con arguments) for accepting or rejecting a controversial standpoint [17]. In particular, we are interested in modelling the process of decision-making by identifying reasons for a decision and methods to analyse relationships between conflicting issues and establish how the agreement has been achieved. We believe that argument-based practical reasoning, in particular, is suited in the BIM context, as it focusses specifically in the study of how to decide on a course of action in order to manage interdependencies and avoid conflicts with others' commitments [18].

In this paper, we propose an initial study to understand the potential of argumentation-based reasoning in analysing the difficulties that arise during the collaborative reasoning process of early design stages. The support is provided via a graphical representation of arguments previously used for argument analysis (e.g., [19]), combined with autonomous support for identification of supported design solutions through argumentation-based reasoning [20]. This approach maps different solutions, and supporting and conflicting relationships between alternatives. This is a systematic method to record important elements of the reasoning processes involved in the stages of identification of design solutions highlighting the fundamental pieces of information, requirements and constraints that underpin this decision, and what alternative solutions were considered to improve future design. This method applied in large scale studies, such as in [1], would also be helpful to inform elaborations of the CDS-Protocol by identifying issues discussed in the process that could be further protocolled to improve the effectiveness of collaborative teamwork and avoid controversial and timeconsuming discussions.

\section{Research context: conflicts during collaborative design}

This paper is focusing on obstacles that occur during collaborative design processes, especially issues with informed collaboration, identification of argumentation workflow and careful documentation of disputes during collaborative design. Quite often collaborative design is hindered by heavy reliance on expensive and time consuming processes, poor incorporation of some important design concerns (typically later life-cycle issues such as environmental impact), as well as reduced creativity due to the tendency to incrementally modify known successful designs rather than explore radically different and potential superior ones [21, pp. 201]. Furthermore, and according to Randy [22], obstacles that 
may appear during the collaborative design process are issues of workflow, education and different design and engineering backgrounds of the professionals, technological challenges arising with different types of software, team working, cost and responsibility. These issues can be further distilled into misunderstandings and failures of cognition distribution that lead to problems within teams and projects that could be successfully tackled by establishing a shared understanding for harmonic communication.

\subsection{Errors during collaborative design}

Errors limit a task performance and can be costly; on the other hand errors have potentials of informing about problems within organisations, they promote learning by making professionals adapt to changes and they can reveal issues within processes that were considered standardised [23]. Errors can arise from the interactions between members of design teams, professionals and the use of technological tools and professionals and formal organisation, as reported by Busby [23]. These errors among professional interactions occur due to misinformation and miscommunications, like failure to involve relevant professional bodies, not informing about problematic situations and effects of different design actions and verifying decisions. Additional reasons include lack of projects scope definition and strategies information according to the involved stakeholders, and lack of understanding in the design processes among different professionals. Further failures appear when professionals interact with design representations and involve misuse of design features and conventions, lack of suitable review of the designs, problems with use of appropriate software, lack of relevant guidance for occasional users and no feedback for adapting software use according to issues previously occurred. What is more, errors that are the result of interactions between professionals and organisations/practices or with external environment might result due to incorrect work allocation and due to mistakes with work conventions for the required activities. The absence of suitable professionals for tackling the appropriate design problems and of notification mechanisms for changes in plans and designs can also lead to errors and mistakes. Eventually, complications with involved professionals on their relevant task goals combined with lack of planned synchronisation are also potential problems for prompting errors during design processes.

\subsection{Conflicts during collaborative design}

Conflicts are an additional important issue during collaborative design, due to the socio-technical dynamics and interactions that affect projects progress [24]. Collaborative design encompasses a deep human aspect where objectification is not always achieved; neither is decisions transparency and removal of judgmental elements among team members, thus resulting in conflicts among team members. As a result, conflicts within a team undertaking a project of the built environment could be a rather expensive issue, since it can potentially lead to delays and/or 
terminations of collaborations, a costly problem of the AEC industry [25]. High costs for changing partners and apprehension of clauses within contracts for legal sanctions are additional consequences of conflicts. Subsequently, effective conflicts management is essential during collaborative design and the clash of ideas can actually promote ideas generation, especially during the early design stages.

Conflicts identification and management can achieve insight and information among the involved parties regarding the core of the project; it can create a cooperative context between the participants and re-build the relationships on a new constructive basis by bridging the gaps between the different perceptions of the involved stakeholders [25]. The ways that can be achieved incorporate identification of conflictual events and transparent analysis of different perceptual ideas about the project. Conflicts management initiates with the identification of the issues that led to the conflicts, either by interviewing the different participants or by data resources, while conflicts can be interpreted according to perceptions and processes of the involved sides. The next step would initiate with assessing these differences in opinions according to projects governance mechanisms, which are case dependant and might include among others mechanisms of incentives, authority and trust. These mechanisms could also be comprised of formal aspects like contracts, official and unofficial agreements, patterns of behaviour, organisational procedures and informal aspects like trust and ease of adaptation.

\subsection{Handling conflicts via argumentation-based reasoning}

Modelling how decisions are made in a collaborative design process is a complex task as there are a lot of interdependent issues that lead to a solution. Computational models of argumentation have the potential to make this process more transparent, allowing a retrospective understanding of how conflicts were resolved.

Argumentation theory has increasingly received attention in Artificial Intelligence as a mechanism to represent autonomous reasoning under incomplete information [17], by providing methods for identifying acceptable arguments and their supported conclusions. An argument is considered accepted if its supporting arguments (i.e., pros) are defended against attacking arguments (i.e., cons). Arguments represent defeasible logical inferences; consider argument $A_{1}$, "We should elevate the building on stilts to control humidity" (adapted from a study of Leon [1]). An attack is an argument $A_{2}$, "An elevated structure will reduce the ability to access the building; hence, we should not elevate the building". The elevation of the building cannot be accepted since $A_{2}$ attacks $A_{1}$. However, in the light of new received information the conclusions may be reconsidered. If $A_{2}$ is attacked by a new argument $A_{3}$, "The building is on a slope and a ramp may lead to the entrance, which will not impede the access", claim $A_{1}$, defended by $A_{3}$, may be reinstated.

The potential of argumentation for design purposes has been discussed in Fischer et al. [26], showing that solutions to the design tasks are dependent upon the argumentative discussion over design issues. Extraction and identification of 
arguments have also been considered in previous research to support the analysis of discussions between professionals. Stumpf and McDonnell [27] employed schemes (i.e., patterns of reasoning) to model shifts on the problem framing. Both studies, however, do not consider a computational model of argumentation, rather a method to record positive and negative reasons for a solution. In a more recent study, Baroni et al. [28] building upon [26] proposed a system to estimate the level of support that each solution received by different designers. These methods examine problems based on matrix-based resolution processes and are mainly geared towards the identification of a solution scored against a number of criteria.

In this research, we are interested in analysing the reasoning process, without constraining the core creative phase of design, where new information and alternative solutions developed in collaboration are continuously added to the process. For this purpose, we use of argument diagramming [19], a method to construct a graph of boxes representing premises and conclusions of arguments and arrows representing pro/con relationships among arguments in order to show an overview of the chain of reasoning among AEC professionals. The resulting map is evaluated via a computational argumentation model to suggest supported conclusions [20]. This analysis makes the decision process more transparent highlighting reasons for conflicting opinions between professionals so as to inform future decisions.

\section{Methodology}

During this research, we analysed a number of segments from the two studies that have been used to test the effectiveness of the CDS Protocol [1]. The studies focused on testing the collaborative design processes during feasibility and concept stages involving multidisciplinary design teams that developed a design concept for an educational office building. The participants in both studies were design professionals with experience in the AEC industry, including two to three architects, a quantity surveyor, a building surveyor, a structural engineer and a construction manager. In the first study professionals followed an unstructured conceptual design process, while the second study was performed following the recommended CDS Protocol, thus making use of a managed facilitation process throughout the design project. Our objective is to further analyse the resolution of conflicts in these studies via the methodology presented in this section. This analysis supports new elaborations of the CDS Protocol by identifying stages where more controversial issues arise. Our analysis may also be accessed by professionals, in the form of a graph, to inform the subsequent stages of the design process.

Our methodology involves three phases: data extraction and transcription, argument diagramming and argument evaluation. This has been developed with a preliminary study of the segment of discussion from the analysis of [1] (Table 1).

The data extraction and transcription phase is focused on the identification of stages of collaborative design that involve debate over options as well as creation of new solutions. The studies raw data are collected according to protocol analysis 
as described in [1]; the studies are video recorded and divided into smaller units (segments). These segments are divided according to subjects' intention and to the theme of the content $[29,30]$. In the studies, each segment includes a description of what is happening together with the applied actions' codings. The coding categories include collaboration actions, perceptual and conceptual actions, and physical actions. In Table 1 we only report the action description used to model the reasoning process; we will consider dialogical aspects in future research.

The argument diagramming phase consists of identifying sentences that represent premises and conclusions of an argument. The graph format employed is that of [20] where the graph explicitly shows the kind of relationships between these sentences and can be easily translated into a formal model of arguments.

Definition 1 An argument graph consists of a set of nodes that contains sentences $p_{i}$, a set of link nodes of type Pro and Con indicating a supporting and conflicting link respectively, and a set of edges that connect nodes.

An edge of the graph is represented textually as $\mapsto$. In the figures nodes are boxes, pro-links are "+" circles and con-links are "-" circles. An argument is of the form $A_{1}:\left[p_{a}, \ldots, p_{m} \mapsto\right.$ Pro $\left.\mapsto p_{r}\right]$ composed by a Pro-link that has $p_{a}, \ldots, p_{m}$ as incoming nodes, premises of $A_{1}$, and an outgoing node $p_{r}$, conclusion of $A_{1}$. A Con-link is an attacking relationship between arguments.

Definition 2 Given $A_{2}:\left[p_{b}, \ldots, p_{n} \mapsto\right.$ Pro $\left.\mapsto p_{q}\right], A_{1}$ attacks $A_{2}$ if the conclusion of $A_{1}$ conflicts with: the conclusion of $A_{2},\left[p_{r} \mapsto\right.$ Con $\left.\mapsto p_{q}\right]$ or conflicts with a premise of $A_{2},\left[p_{r} \mapsto\right.$ Con $\left.\mapsto p_{i}\right]$ and $p_{i} \in\left\{p_{b}, \ldots, p_{n}\right\}$.

In the argument evaluation phase, a method to map such graph to an argumentation framework and extract sets of acceptable arguments is employed. The objective is to identify in an automatic way a node that contains a sentence that may or may not be supported. In the figures, we will indicate supported sentences with "V" and non supported sentences with "X". Assume that a set of acceptable arguments in the mapped argumentation framework is $\Pi_{j}=\left\{A_{a}, A_{b}, \ldots\right\}$.

Definition 3 Given $\Pi_{j}=\left\{A_{a}, A_{b}, \ldots\right\}$, a function eval $\left(p_{i}\right)$ returns an assignment for a sentence $p_{i}$ such that: $\operatorname{eval}\left(p_{i}\right)=V$ (supported), if $p_{i}$ is a conclusion of $A_{k} \in \Pi_{j}$; eval $\left(p_{i}\right)=X$ (unsupported) otherwise.

In the analysis, a supported sentence indicates an accepted solution, or a reason for a solution to be accepted.

\section{Study}

In this section, we describe the results of the analysis performed according to our methodology of two segments of discussion proposed in [1].

In Figure 1 we show the argument graph extracted from the segment in Table 1. We may observe a typical instance of practical reasoning [18], where at first a solution (i.e., large quantity of windows) is proposed, then analysed via the chain of arguments on the left-hand side of the graph, and finally refined with an alternative solution (i.e., reduced number of windows in certain rooms). The automatic evaluation is shown on top of each box where, in this case, the labelling 
Table 1: Glazing dialogue. Participants: Architect 1 and 2 (A1/A2); Project Manager (PM); Quantity Surveyor (QS).

A1: Comments about the materials, adds that they all agree about their preference to large glazing-covered areas and Nordic design inspiration

A2: Argues that too many windows might be a problem

PM: Adds that too much glazing can cost a lot and might cause problems to the construction

A1: Replies that you can shadow it, thus providing solutions

PM: Talks about problems with glare

A2: States that some rooms can have controlled shading while others can be more or less glazed depending on the heating loads and working needs.

QS: Agrees and further comments on it

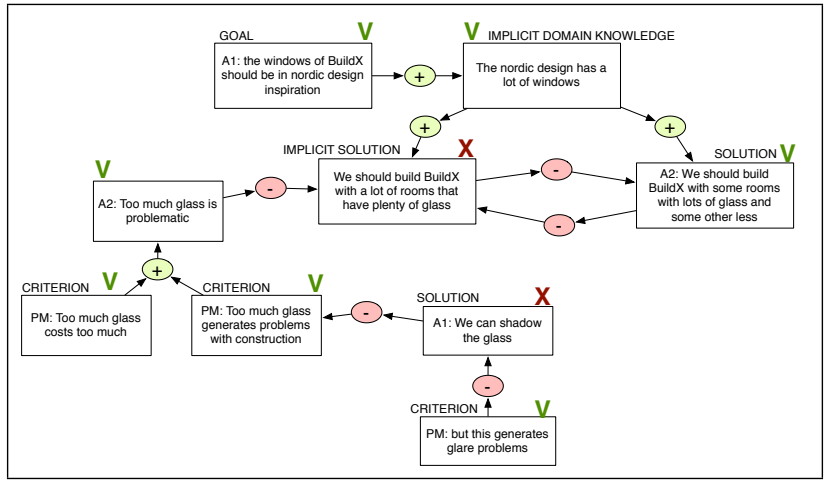

Figure 1: Glazing argument graph.

corresponds to the decision that the designers have reached suggesting that our approach may be suitable for analysing the decision-making process.

An initial distinction between type of sentences used to evaluate different solutions may also be identified. These sentences correspond to elements derived from practical reasoning schemes such as goals, actions (i.e. design solutions) and values (i.e. design criteria) [18]. We have included additional nodes to make explicit the common domain knowledge (i.e., a nordic design requires a lot of windows), and the inferences that led to the discussion of the lefthand side solution (i.e., windows require a large quantity of glass). These nodes act as unstated premises and conclusions that are necessary to comprehend the connections between the design goal and the solutions. The initial set of type of sentences for design may be classified as: domain knowledge often implicit; design solutions - in the graphs, they are identified with sentences from practical reasoning for actions, e.g. "We should do ..."; design criteria - used to comment on choices; and design goals. 


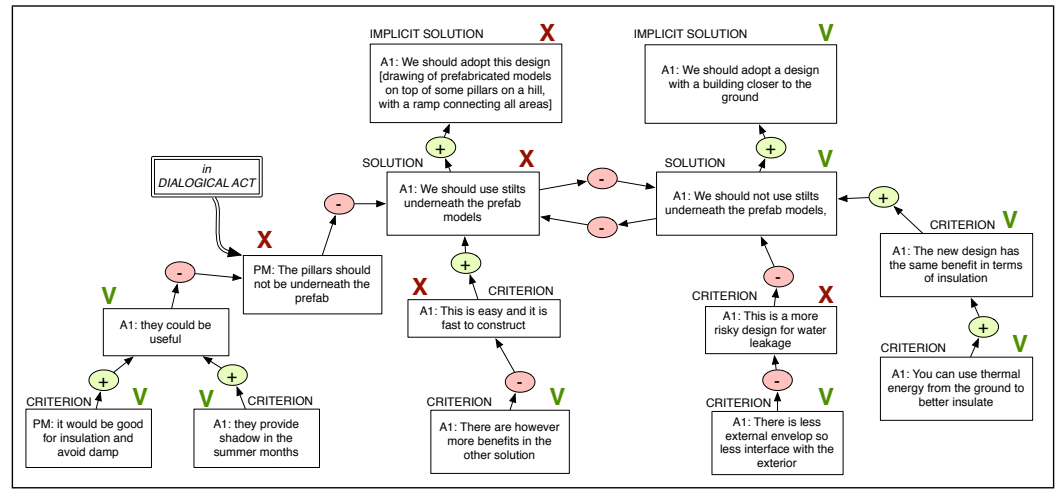

Figure 2: Pillars argument graph.

The study of how preferences among criteria led to a decision is out of the scope of this initial research. This interesting aspect will be further investigated in future research as the discussion among professionals is often interleaved with seeking agreement over those criteria. The annotation of type of sentences in combination with labelling supported argument may offer a clearer analysis of what kind of knowledge has contributed to the resolution of conflicts towards an agreement on a design solution. For example, a solution with a large quantity of windows is deemed not acceptable due to issues in handling too much glass, glaring and costs.

The graph in Figure 2 represents the chain of reasoning on a second segment, which is an extended version of the dialogue presented in Table 1. As in the previous study, we may observe the presence of similar types of sentences and different solutions. In this segment, we see some intent of architect A1, who is trying to lead the design, to persuade the other participants. A1 moves a number of defences in support of the use of pillars: by suggesting a reason for accepting it (e.g., it is easy to construct); by attacking and defending it to prevent a refusal of the initial design; and by responding to a question that challenges the design (the sequence on the left-hand side). Dialogical acts will be subject of future studies as they are fundamental to better analyse interpersonal reasoning. Interestingly, we may also notice the collaborative attitude of the professionals, where an antagonist such as the PM changed role and complemented A1's defence for the challenged solution, in relation to the building sustained by pillars being suitable for insulation.

\section{Discussion}

The technological advances promoted with the application of BIM enable simulations of the built environment projects, from the concept stages, which is the focus of the research in [1], to the cost, constructability, and time and site organisation of the projects. However, the possibility of mastering the diversity of technical languages and seeking the multiplicity of professional viewpoints 
from the early design stages can produce informed results that are less prone to errors and costly design iterations at later and more advanced stages. An informed and transparent collaboration and communication within the AEC industry can promote understanding and solution finding, as it is showcased in this paper. Additionally, the behavioural parameter is an issue to the collaboration processes between the multidisciplinary design teams and, as a result, the design process is critically affected by issues of communication, social processes, negotiation and reflection. Consequently, a rigorous monitoring and analysis of the decision steps through argumentation processes could provide valuable clarity to the decision making process, prevent construction related legal disputes and eventually support multidisciplinary collaborative team work, thus smoothing and promoting collaborative BIM.

In future research, we will explore characteristics of the discussion that may need further formalisation to improve our method of analysis, considering for example the introduction of argument schemes [27]. Further research may focus on argument mining, a method to extract arguments from text that may allow us to study collaborative design processes using our methodology in large-scale studies.

\section{References}

[1] Leon, M., Development of a computer mediated multidisciplinary design protocol and its application to the early architectural design stages, 2015.

[2] Wang, L., Shen, W., Xie, H., Neelamkavil, J. \& Pardasani, A., Collaborative conceptual design-state of the art and future trends. Computer-Aided Design, 34(13), pp. 981-996, 2002.

[3] PAS1192-2:2013, Specification for information management for the capital/delivery phase of construction projects using building information modelling. Technical report, British Standards Institution, 2013.

[4] Cross, N., Engineering Design Methods - Strategies for Product Design. John Wiley \& Sons: West Sussex, England, 4th edition, 2008.

[5] Pahl, G. \& Beitz, W., Engineering design: a systematic approach. Springer: Berlin, Germany, 2nd edition, 1995.

[6] Daenzer, W.F., Systems Engineering. Industrielle Organisation Zürich: Zurich, revision edition, 2002.

[7] Rittel, H., Second-generation design methods. Developments in Design Methodology, pp. 317-327, 1984.

[8] Fischer, G., Symmetry of ignorance, social creativity, and meta-design. Knowledge-Based Systems, 13(7-8), pp. 527-537, 2000.

[9] Dainty, A., Moore, D. \& Murray, M., Communication in Construction. Taylor \& Francis: Oxon, 2006.

[10] Penny, R.K., Principles of engineering design. Postgraduate medical journal, 46(536), pp. 344-349, 1970.

[11] Schön, D., The Reflective Practitioner: How Professionals Think in Action. Ashgate Publishing Limited: London, 1991. 
[12] Rod, M., Subjective personal introspection in action-oriented research. Qual Research in Orgs \& Mgmt, 6(1), pp. 6-25, 2011.

[13] East, E.W., Construction operations building information exchange, 2013.

[14] BS 7000-4:2013, Design management systems. guide to managing design in construction. Technical report, British Standards Institution, 2013.

[15] Sinlair, D., (ed.) RIBA Plan of Work 2013. RIBA: London, 2013.

[16] Leon, M., Laing, R., Malins, J. \& Salman, H., Development and testing of a design protocol for computer mediated multidisciplinary collaboration during the concept stages with application to the built environment. Procedia Environmental Sciences, 22, pp. 108-119, 2014.

[17] Maudet, N., Parsons, S. \& Rahwan, I., Argumentation in multi-agent systems: Context and recent developments. Argumentation in Multi-Agent Systems, Springer Berlin Heidelberg, volume 4766 of Lecture Notes in Computer Science, pp. 1-16, 2007.

[18] Atkinson, K. \& Bench-Capon, T., Practical reasoning as presumptive argumentation using action based alternating transition systems. Artificial Intelligence, 171(10-15), pp. 855-874, 2007.

[19] Reed, C. \& Rowe, G., Araucaria: Software for argument analysis, diagramming and representation. International Journal on Artificial Intelligence Tools, 13(04), pp. 961-979, 2004.

[20] Toniolo, A., Norman, T.J., Etuk, A., Cerutti, F., Ouyang, R.W. et al., Supporting reasoning with different types of evidence in intelligence analysis. In Proceedings of the Fourteenth International Conference on Autonomous Agents and Multiagent Systems, 2015.

[21] Klein, M., Sayama, H., Faratin, P. \& Bar-Yam, Y., The dynamics of collaborative design: Insights from complex systems and negotiation research. Concurrent Engineering, 11(3), pp. 201-209, 2003.

[22] Randy, D., Working With Others In BIM, John Wiley \& Sons: New Jersey, chapter 4, pp. 91-126. BIM and Integrated Design: Strategies for Architectural Practice, 2011.

[23] Busby, J.S., Error and distributed cognition in design. Design Studies, 22(3), pp. 233-254, 2001.

[24] Lu, S.C.Y., Cai, J., Burkett, W. \& Udwadia, F., A methodology for collaborative design process and conflict analysis. CIRP Annals Manufacturing Technology, 49(1), pp. 69-73, 2000.

[25] Vaaland, T.I., Improving project collaboration: start with the conflicts. International Journal of Project Management, 22(6), pp. 447-454, 2004.

[26] Fischer, G., Lemke, A.C., McCall, R. \& Morch, A.I., Making argumentation serve design. Human-Computer Interaction, 6(3-4), pp. 393-419, 1991.

[27] Stumpf, S. \& McDonnell, J., Talking about team framing: using argumentation to analyse and support experiential learning in early design episodes. Design Studies, 23(1), pp. 5-23, 2002.

[28] Baroni, P., Romano, M., Toni, F., Aurisicchio, M. \& Bertanza, G., Automatic evaluation of design alternatives with quantitative argumentation. Argument \& Computation, 6(1), pp. 24-49, 2015. 
228 Building Information Modelling (BIM) in Design, Construction and Operations

[29] Suwa, M., Purcell, T. \& Gero, J., Macroscopic analysis of design processes based on a scheme for coding designers' cognitive actions. Design Studies, 19(4), pp. 455-483, 1998.

[30] Gu, N., Kim, M.J. \& Maher, M.L., Technological advancements in synchronous collaboration: The effect of 3D virtual worlds and tangible user interfaces on architectural design. Automation in Construction, 20(3), pp. 270-278, 2011. 